(A) Check for updates

Cite this: Food Funct., 2019, 10, 4751

\section{A single-enzyme system for starch digestibility screening and its relevance to understanding and predicting the glycaemic index of food products $\dagger$}

\author{
Cathrina H. Edwards, (D) * Noriane Cochetel, Lauren Setterfield, Natalia Perez-Moral \\ and Frederick J. Warren (D)
}

\begin{abstract}
There is currently great interest in increasing provisions of healthier carbohydrate foods, particularly those that possess a low Glycaemic Index (Gl) when measured in vivo. The metabolic response to many starchrich foods is driven largely by differences in the rate and extent of starch amylolysis. Enzyme-kinetic parameters obtained from high-throughput in vitro amylolysis assays therefore have potential for rapid prediction of Gl for starch-rich foods. The aim of this study was to evaluate the usefulness of a starch digestibility screening method and resulting enzyme-kinetic parameters in comparing and predicting the Gl of a range of carbohydrate-rich foods. Starch-rich foods $(n=20)$ with $\mathrm{Gl}$ ranging from 36 to 81 were digested by porcine pancreatic $\alpha$-amylase for 90 min under a fixed enzyme-substrate ratio ( $4 \mathrm{U} / 10 \mathrm{mg}$ starch) at $37{ }^{\circ} \mathrm{C}$ on a rotary mixer. Starch digestion progress was determined by quantification of reducing sugar concentration in aliquots collected throughout the incubation. Indices of starch digestibility $\left(C_{20}, C_{60}\right.$, $C_{90}, \mathrm{HI}, C_{\infty}$, and $k$ ) were obtained and compared with $\mathrm{Gl}$ values. Digestibility curves revealed differences in the starch amylolysis for the broad range of foods tested. In vitro starch digestibility indices were significantly correlated $(p<0.01)$ with $\mathrm{Gl}$, with the exception of the rate constant, $k$. Out of all the indices tested, $C_{90}$ and $C_{\infty}$ were the most strongly correlated with in vivo rankings for $G$ l of matched food products ( $T_{\mathrm{b}}=0.596, p<0.001$ and $T_{\mathrm{b}}=0.599, p<0.01$, respectively), however the digestibility plots obtained for some of the more slowly digested foods were linear over 90 min meaning that $C_{\infty}$ and $k$ could not be obtained from first order kinetic analysis. $C_{90}$ was most strongly correlated with the absolute Gl values $(r=$ $0.724, p<0.001$ ). Overall starch digestibility profiles reflected differences in starch amylolysis for food with varying $\mathrm{Gl}$, and $C_{90}$ provided the best indication of absolute and relative $\mathrm{Gl}$ values across all product categories. The in vitro starch digestibility screening method shows potential for rapid prediction of Gl values and is recommended for early stage food product development and for mechanistic studies.
\end{abstract}

Received 22nd March 2019, Accepted 20th May 2019 DOI: $10.1039 /$ c9fo00603f rsc.li/food-function

\section{Introduction}

Starch-rich foods are a major source of dietary energy and therefore play an important role in influencing public health. The metabolic responses to starch-rich foods can be vastly different and are a critical consideration for the design of healthier foods. For instance, it has been shown that consumption of starch-rich foods with a low Glycaemic Index (GI) and high amounts of Resistant Starch (RS) are associated with reduction in Type 2 Diabetes and Cardiovascular Disease risk and could play an important role in the dietary management of these diseases. ${ }^{1}$ Given the alarming prevalence of chronic diet-related

Quadram Institute Bioscience, Norwich Research Park, NR4 7UA Norwich, UK. E-mail: Cathrina.edwards@quadram.ac.uk; Tel: +44 (0)1603251466

$\dagger$ Electronic supplementary information (ESI) available. See DOI: 10.1039/ c9fo00603f diseases, increasing the supply of consumer-acceptable carbohydrate foods that possess these (and other) desirable properties should be a priority. Although in vivo studies remain the gold standard for testing physiological outcomes, in vitro methodologies play an important role in rational product design.

The in vivo 'Glycaemic Index methodology' for ranking carbohydrate foods according to the glycaemic response that they evoke is well-established ${ }^{2}$ and many foods have already been indexed. ${ }^{3}$ The resource-demanding nature of human studies does however impose some restriction on the number of food products that can be tested, and inter- and intra-individual variations (although relevant) means that human studies lack the sensitivity to detect subtle changes that may be of importance to product concept development. Thus, highthroughput in vitro methodologies that rank products by predicted GI would aid rational design of interventions for in vivo 
testing while providing for additional mechanistic insight to inform formulation of functional food products.

The glycaemic response to starch-rich food is strongly influenced by the rate and extent of starch digestion by $\alpha$-amylase in the upper gastrointestinal tract. ${ }^{4}$ In vitro methods that simulate digestive conditions can therefore potentially provide a means of predicting relative differences in GI of various food. Digestion models that replicate the biochemical conditions (enzymes, electrolytes, $\mathrm{pH}$, mixing etc.) of oral, gastric and intestinal digestion $^{5,6}$ are increasingly popular, however the methodological complexity and large number of reagents required prevents the use of these models for high-throughput screening. Moreover, for predicting the glycaemic response to starch-rich foods, this level of complexity may not always be required; indeed, good agreement between in vitro starch hydrolysis indices and glycaemic responses has been achieved previously using simpler protocols. ${ }^{7,8}$ One limitation of these previous methods however is the tendency to pulverise, grind or homogenise foods prior to analysis; such treatments breakdown food structures (such as plant cells, tissues, intracellular networks) that are now known to be of great importance with regard to influencing digestion kinetics and measurement of Type 1 resistant starch (RS1) (i.e. starch that is resistant to digestion because it is physically inaccessible to digestive enzymes). ${ }^{9-11}$ It is therefore important that in vitro tests are assessed based on their performance across a broad range of food products with different structures and susceptibility to digestion.

We have previously reported on the value of an enzymekinetic approach that is routinely used in our laboratory for mechanistic digestibility studies of starch-rich food materials. ${ }^{12,13}$ When combined with a controlled and mechanistically-informed approach to sample preparation, this methodology may enable rapid comparison of starch susceptibility to digestion and prediction of GI in a range of carbohydrate foods. The aim of the present study was to apply this in vitro starch amylolysis methodology to a broad range of food products and evaluate the use of in vitro starch digestibility indices for rapid prediction of GI values.

\section{Materials \& methods}

\section{Selection and preparation of food materials}

Commonly consumed starch-rich foods were selected from various product categories (pulses, tubers, cereals, pasta, rice, bread and biscuits) to cover a range of Glycaemic Index (GI) values, as reported by Atkinson et al. 2008. ${ }^{3}$ An overview of the food products, starch content and method of preparation is provided in Table 1 and further details of food composition is provided in OSM1. The nutrient composition data was obtained from nutrition declaration information provided on food products (according to EU regulation no. 1169/2011) with the exception of two products where the on pack information was not representative of how the product was prepared, and therefore food composition reference values were used. ${ }^{14}$ The starch content was calculated by subtracting sugars from the available carbohydrate value. Values reported on an 'as weighed' basis were corrected to account for the moisture content (determined on a Moisture Balance, VWR International) at the time of weighing for digestibility analysis. It is noteworthy that large errors are commonly observed for calculated and direct enzymic determination of total starch, and that this is a likely source of error in both the in vitro assays and the in vivo studies. Wherever possible, the source and method of food preparation for in vitro analysis matched the description provided in the Glycaemic Index tables, and any discrepancies are disclosed within these tables.

In preparation for in vitro testing, the food samples were freshly cooked (if applicable) according to manufacturer instructions and ground to achieve a controlled particle size reduction. In general, dry products (i.e., biscuits, bread, breakfast cereals) were ground to 'crumbs' and sieved to select a standard particle size fraction $(250-500 \mu \mathrm{m})$. This size range was chosen as it provides a reasonable yield of representative particles in which a high proportion of the starch granules are exposed on the particle surface. Cooked products were prepared according to manufacturer instructions and analysed immediately (within $30 \mathrm{~min}$ ) of cooking. Canned products were drained and then ground to a loosely formed paste. For this particular study, these conditions were selected in an effort to preserve food structures (e.g., plant cells and RS1) that are known to reach the intestinal lumen. Variables such as particle size, cooking and cooling conditions, were standardised and controlled to enable good reproducibility.

\section{Starch digestibility assay}

The principles of the starch digestibility assay have been described previously. ${ }^{12,13}$ The method is described below and is similar to that performed by Edwards $2018,{ }^{15}$ with minor modifications to the volumes and time-points used. An important feature of this procedure is that the starch-amylase ratio has been kept constant for all food products tested such that any differences observed reflect the starch digestibility of the food rather than their variable starch contents.

In brief, freshly prepared food materials (see section 2.1 for source and preparation) were weighed into $15 \mathrm{~mL}$ corning tubes and suspended in $10 \mathrm{~mL}$ phosphate buffered saline (PBS, pH 7.4) so that each tube contained $100 \pm 2 \mathrm{mg}$ starch. Sample tubes were mixed for $20 \mathrm{~min}$ at $37^{\circ} \mathrm{C}$ on a Stuart SB3 rotary mixer (20 rpm, 30 angle) inside an incubator (E24 Excella, New Brunswick Scientific) to equilibrate. Before starting the assay, a $200 \mu \mathrm{L}$ 'blank' aliquot of each sample was taken into a $1.5 \mathrm{~mL}$ microcentrifuge tube containing $200 \mu \mathrm{L}$ 'stop solution' (0.3 $\mathrm{M} \mathrm{Na} \mathrm{CO}_{3}, \mathrm{pH}$ 9). The $0.3 \mathrm{M} \mathrm{Na} \mathrm{CO}_{3}$, referred to as 'stop solution', inactivates the amylase activity in the aliquots and is used to promptly stop the amylolysis at each time point. To start the assay, porcine pancreatic $\alpha$-amylase (EC 3.2.1.1 supplied in DFP-treated suspension of $2.9 \mathrm{M} \mathrm{NaCl}$ containing $2 \mathrm{mM} \mathrm{CaCl}_{2}, \mathrm{~A}_{255}$, Sigma-Aldrich Co. Ltd, Poole, UK) prepared in PBS ( $\mathrm{pH} 7.4$ at $37^{\circ} \mathrm{C}$ ) was added to achieve an activity of $4 \mathrm{U} \mathrm{mL}^{-1}$ in the digestion mixture (i.e. 
Table 1 Source and preparation of food materials

\begin{tabular}{|c|c|c|c|}
\hline Sample ${ }^{a}$ & Method of preparation & $\begin{array}{l}\text { Starch } g \\
\text { per } 100 \mathrm{~g}^{b}\end{array}$ & Comparator $^{e}$ \\
\hline \multicolumn{4}{|l|}{ Biscuits } \\
\hline $\begin{array}{l}\text { Fullkorn digestive (United } \\
\text { Biscuits, Sweden) }{ }^{d}\end{array}$ & $\begin{array}{l}\text { Ground with pestle and mortar and } \\
\text { sieved to collect } 0.25-0.5 \mathrm{~mm} \text { particles }\end{array}$ & 39.0 & $\begin{array}{l}\text { Average of Digestives (UK) and Digestives (Canada). } \\
\text { REF 630; } 631\end{array}$ \\
\hline $\begin{array}{l}\text { Water cracker (United Biscuits, } \\
\text { UK) }\end{array}$ & & 72.3 & $\begin{array}{l}\text { Average of water cracker (Canada) and water } \\
\text { cracker (Arnotts, AU). REF 740; } 741\end{array}$ \\
\hline $\begin{array}{l}\text { Cornish wafer (United Biscuits, } \\
\mathrm{UK})^{d}\end{array}$ & & 55.7 & $\begin{array}{l}\text { Average of Cream cracker (LU Triumfo, Brazil), and } \\
\text { Jatz plain salted cracker biscuits (Arnotts, AU). REF } \\
718 ; 720\end{array}$ \\
\hline Oatcake (Nairn's UK) & & 55.5 & $\begin{array}{l}\text { Average of Oat biscuit (UK); (China); Nairn's } \\
\text { ginger; Oatmeal (Canada); Highland Oatmeal } \\
\text { (Weston, AU). REF 676; 677; 678; 679; } 641\end{array}$ \\
\hline Rich tea biscuit (Lyons, UK) & & 53.4 & $\begin{array}{l}\text { Average of Rich Tea (UK) and Rich Tea (Canada). } \\
\text { REF 699; } 700\end{array}$ \\
\hline
\end{tabular}

\section{Breads}

Bread; sunflower \& barley (Vogel's Blended into bread crumbs

\section{UK)}

Bread; wholemeal (Tesco, UK)

Bread; white (Tesco, UK)

Ground with pestle and mortar and sieved to collect $0.25-0.5 \mathrm{~mm}$ particles

Supermarket Ltd, UK)

Balance (Sainsbury's

Supermarket Ltd, UK)

\section{Pasta}

Spaghetti (Napolina)

\section{Potatoes}

King Edward Potato (Sainsbury's Supermarket Ltd, UK)

Charlotte potatoes (Sainsbury's

Supermarket Ltd, UK)

\section{Pulses}

Chickpea-canned in water

(Sainsbury's Supermarket Ltd,

UK)

Brown Lentils-canned in water

(Napolina)

Butter beans-canned in water

(Sainsbury's Supermarket Ltd,

UK)

\section{Rice}

Long grain white rice-easy cook, parboiled (Sainsbury's

Supermarket Ltd, UK)

\section{Vegetables}

Sweet potatoes (Sainsbury's

Supermarket Ltd, UK)

Garden peas-frozen (Birds Eye)
Peeled, cut, added to boiling water, covered and simmered 15-20 min moderate heat, mashed

16.7

Drained, ground with pestle and mortar

Added to boiling water, 15-18 min moderate heat, drained well, ground with pestle and mortar

Peeled, cut, added to boiling water, covered and simmered 8-10 min moderate heat, mashed Boiled from frozen; added boiling water to cover, covered and simmered $3 \mathrm{~min}$, ground and blended
34

Sunflower and barley bread (Vogel's, UK). REF 111

Mean of 10 studies on wholemeal (whole wheat) wheat flour bread. REF 234-243

Mean of 16 studies on white wheat flour breads. REF 172-187

Mean of five studies on cornflakes. REF 321-325 Rice Pops ${ }^{\mathrm{TM}}$ (Sainsbury's, UK), with semi-skimmed milk. REF 468

Balance $^{\mathrm{TM}}$ breakfast cereal (Sainsbury's, UK). REF 304

Boiled in $0.7 \%$ salted water for $11 \mathrm{~min}$. REF 1375

King Edward potato, peeled, quartered, boiled 15 min in unsalted water (UK). REF 1639 Charlotte, peeled, quartered, boiled 15 min (UK). REF 1634

Chickpeas, canned, drained, Edgell's ${ }^{\mathrm{TM}}$ brand. REF 1096

Lentils, brown, canned, drained, Edgell's ${ }^{\mathrm{TM}}$ brand. REF 1113

Butter beans, canned, drained, Edgell's ${ }^{\mathrm{TM}}$ brand. REF 1089

Long grain, parboiled 10 min cooking time Uncle Ben's, Masterfoods (Belgium). REF 522

8.9 $\quad$ Mean of boiled sweet potato. REF 1684; $1685 ; 1686$; 1692

Pea, frozen, boiled (Canada). REF 1611

${ }^{a}$ Sample details; manufacturer listed in brackets. ${ }^{b}$ Starch content $\mathrm{g}$ per $100 \mathrm{~g}$ as weighed, sourced from nutrition declaration. ${ }^{c} \mathrm{McCance} \&$ Widdowson entries 11-722. ${ }^{d}$ McCance \& Widdowson entries $13-551 .{ }^{e}$ Comparator products with reference numbers ('REF') as listed in Table A1 'Glycemic index and glycemic load values determined in subjects with normal glucose tolerance: 2008' in Atkinson et al. 2008. ${ }^{3}$ 
containing $10 \mathrm{mg} \mathrm{mL} \mathrm{m}^{-1}$ starch). This enzyme-substrate ratio was chosen on the basis of results obtained with previous studies ${ }^{12,15}$ taking into account the range of starch susceptibility to digestion expected for the type of foods included within the study. One unit is defined as the amount of amylase needed to liberate $1.0 \mathrm{mg}$ of maltose from starch in $3 \mathrm{~min}$ at $\mathrm{pH} 6.9$ at $20^{\circ} \mathrm{C}$. Tubes were promptly returned to the mixer in the incubator after addition of amylase and incubated at $37{ }^{\circ} \mathrm{C}$ in the mixer for the duration of the digestion. After 5 , $10,15,20,25,30,45,60$, and $90 \mathrm{~min}$, aliquots $(200 \mu \mathrm{L})$ were collected from the digestion mixture into an equal volume of the stop solution. Aliquots were centrifuged at $15000 \mathrm{~g}$ for 5 min (Haraeus Pico, Thermo Scientific) to exclude any starch remnants and the supernatants stored at $-20{ }^{\circ} \mathrm{C}$ for subsequent analysis of starch amylolysis products.

\section{Reducing sugar analysis (PAHBAH assay)}

The PAHBAH assay ${ }^{16}$ was subsequently used to determine the concentration of reducing sugars produced from starch amylolysis in aliquots recovered at the various time points. Stored supernatants were appropriately diluted (typically $1: 10$ ) in deioinised water, and $100 \mu \mathrm{L}$ of the diluted sample transferred to a $1.5 \mathrm{~mL}$ Eppendorf ${ }^{\circledR}$ safe-lock ${ }^{\mathrm{TM}}$ tube, to which was added $1000 \mu \mathrm{L}$ freshly prepared 'PAHBAH working reagent' (250 mg p-hydroxybenzoic acid hydrazide dissolved in $4.75 \mathrm{~mL}$ of $0.5 \mathrm{M}$ $\mathrm{HCL}$, and made up to $50 \mathrm{~mL}$ with $0.5 \mathrm{M} \mathrm{NaOH}$ ). These tubes were vortex mixed and secured in a Nalgene ${ }^{\circledR}$ floating rack and incubated at $100{ }^{\circ} \mathrm{C}$ for $5 \mathrm{~min}$, then equilibrated for $10 \mathrm{~min}$ at room temperature before transfer to cuvettes and absorbance measurement $(\lambda=405 \mathrm{~nm}$ ) in a spectrophotometer (Biochrom Libra). Standards containing known concentrations of maltose (0-900 $\mu \mathrm{M})$ were prepared in $1.5 \mathrm{~mL}$ Eppendorf ${ }^{\circledR}$ safe-lock $^{\mathrm{TM}}$ and reacted with PAHBAH reagent as described above, and these were included with every analysis. It is noteworthy that this assay can also be performed on a 96-well plate as described in ref. 15. Reducing sugar concentration in each sample was expressed as maltose equivalents by reference to the standard curve.

\section{Data processing}

Data obtained from the PAHBAH analysis was further processed to correct for endogenous reducing sugars present within the sample prior to addition of $\alpha$-amylase.

For ease of interpretation, data expressed in maltose equivalent concentration units was converted to show the corresponding percentage of starch digested during amylolysis according to eqn (1):

$$
(\text { Starch amylolysis } \%)_{t}=\frac{[\text { maltose }]_{t}}{[\text { maltose }]_{\text {substrate }}} \times 100
$$

in which $[\text { maltose }]_{t}$ is the maltose equivalent concentration (after baseline correction) measured in the liquid phase of the reaction mixture at a time point $t$, and [maltose] $]_{\text {substrate }}$ is the theoretical maltose equivalent concentration that would be present at the start of the reaction, assuming that all starch within the food sample can be converted to maltose.
It is noteworthy that the conversion (eqn (1)) assumes that all amylolysis of starch yields maltose, and that this approach does not precisely account for minor products of starch amylolysis (glucose, $\alpha$-limit dextrins and maltodextrins) thereby resulting in a net underestimation of total starch amylolysis. Expressing starch amylolysis relative to a highly digestible, high glycaemic index reference may provide absolute values that relate to GI (see Hydrolysis Index in section 2.5).

\section{Starch digestibility and indices of glycaemic starch}

Starch digestibility curves showing starch amylolysis progress over time were produced for each food material and used to derive the various starch digestibility indices described below and summarised in Fig. 1.

The enzyme-kinetic parameters $C_{\infty}$, which represent the product concentration at the reaction end-point, and $k$, which is the digestibility rate constant, can be used to describe starch amylolysis according to a first-order reaction (eqn (2)). These values were obtained using Logarithm of slope analysis, as described previously. ${ }^{12,13}$

$$
C_{t}=C_{\infty}\left(1-e^{-k t}\right)
$$

in which the $C_{t}$ is the product concentration at a given time, $t$, $C_{\infty}$ is product concentration at the reaction end-point, and $k$ is the digestibility rate constant.

The terms $C_{20}, C_{60}$, and $C_{90}$ represent the extent of starch digested after 20, 60, $90 \mathrm{~min}$ and were obtained from the starch digestibility data. The selection of specific time points for comparison is rather arbitrary, yet this approach has been used previously to describe starch digestibility. In the present study the amylase-starch ratio has been kept constant for all samples to enable direct comparisons.

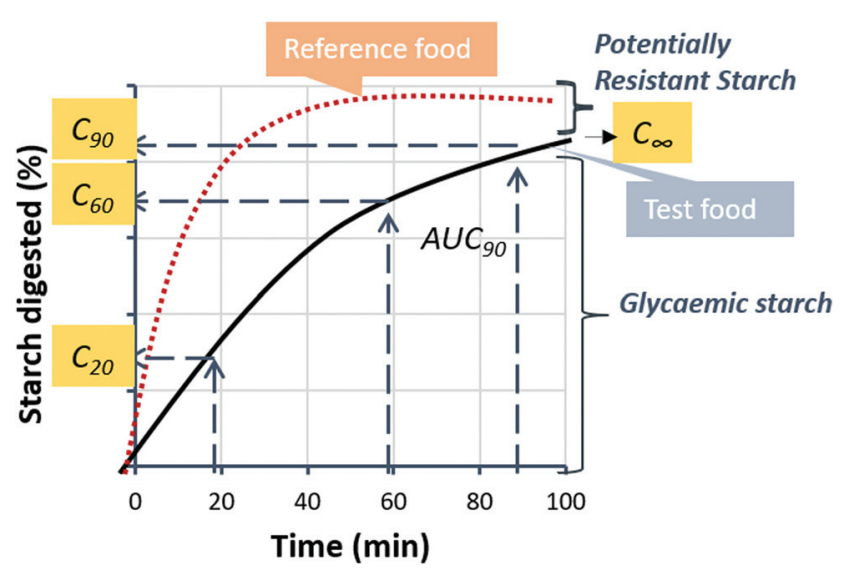

Fig. 1 Schematic of starch digestibility indices. One starch digestibility curve provides for rapid insight into glycaemic and potentially resistant starch fractions. The indices $C_{20}, C_{60}$ and $C_{90}$ correspond to the proportion of starch digested by $\alpha$-amylase after 20,60 and 90 min and are obtained directly from the starch digestibility curve. $C_{\infty}$ is the proportion of starch that is digested at the endpoint of the reaction and is obtained together with the rate constant ( $k$; not shown) by applying logarithm of slope analysis to digestibility data. The incremental area under the curve (iAUC) is used in the calculation of Hydrolysis Index. 
The Hydrolysis index (HI) is the area under the digestibility curve up to $90 \mathrm{~min}$ (iAUC90) of the test sample expressed relative to a highly digestible reference food as shown in eqn (3). In this case, boiled King Edward potato was used as the reference food as this gave consistently the highest starch digestibility value out of all the foods examined.

$$
\mathrm{HI}=\frac{\mathrm{iAUC} 90_{\text {test }}}{\mathrm{iAUC} 90_{\text {reference }}} \times 100
$$

\section{Statistical analyses}

Digestibility curves were plotted using SigmaPlot 14.0 software, and fitted to the first-order equation using non-linear regression. The Area Under Curves was obtained using the Area Below Curves Macro which is available from the Toolbox supplied within the Sigma Plot software. All values are presented as means of assays performed in triplicate with error bars as 95\% CIs or SEM as specified in text and figure legend. In vitro data was compared statistically with published values from in vivo studies of matched food products taken from Atkinson et al. 2008, ${ }^{3}$ using values for healthy subjects with glucose $(\mathrm{GI}=100)$ as the reference. Sources of in vivo data are fully referenced and details of any discrepancies between products are highlighted in the results table (Table 1). Statistical analyses were performed using IBM SPSS Statistics version 22 . Pearson's correlation test (co-efficient denoted $r$ ) was used to test the correlation between absolute GI values and in in vitro indices, whereas Kendalls Tau b (co-efficient denoted $T_{\mathrm{b}}$ ) test was used to test the rank correlation between food ranked based on vitro starch digestibility indices and in vivo Glycaemic Index data.

\section{Results}

Starch digestibility curves are shown for selected products in Fig. 2. Based on GI values ${ }^{3}$ of the food products shown in Fig. 2, the breakfast cereals (Fig. 2A) and breads (Fig. 2B) would be classes as 'high GI (GI $\geq 70$ ) food', rice, spaghetti and charlotte potato (Fig. 2C) would be 'medium GI (GI > 55 and $<70$ ) food' and the pulses (Fig. 2D) would be classed as 'low GI (GI $\leq 55)$ food'. Within each of these categories, there are clear differences in the shape of the digestibility profiles of each food product. For instance, comparison of curves obtained for bread and breakfast cereals (all classed as high GI products) highlights how starch digestibility curves can reveal differences in starch digestion kinetics that are not detected in GI values or other digestibility indices. Food products with the lower GI were digested more slowly and some were still within a linear phase of digestion after $90 \mathrm{~min}$. For these linear digestibility plots (observed for butter bean, chickpea, brown lentil, long-grain rice, sunflower-barley bread, and digestive biscuits), the resulting LOS plots (see example Fig. 3C) were characterised by a low rate constant $(k<0.01)$, with the implication that unreliable $C_{\infty}$ values were calculated (data shown

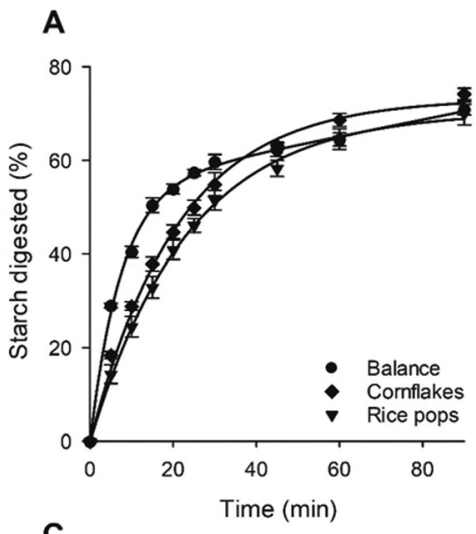

\section{B}

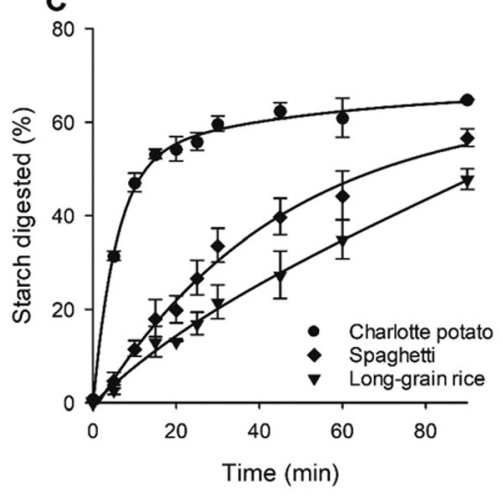

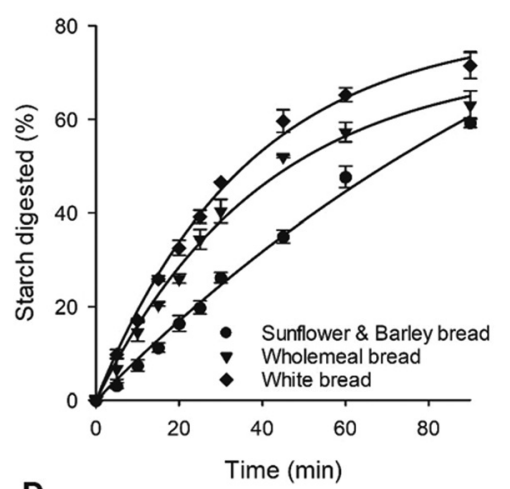

D

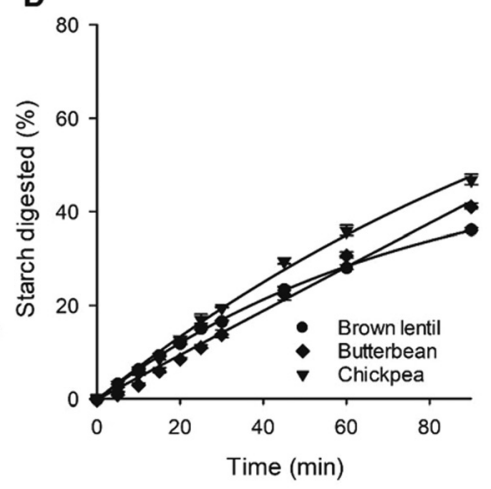

Fig. 2 Starch digestibility curves obtained for breakfast cereals (A); breads (B); potato, spaghetti and rice (C); and pulses (D). Values are means of triplicate analysis with error bars as SEM. Curve fits were obtained by maximum likelihood estimate regression to the first order equation. 
A

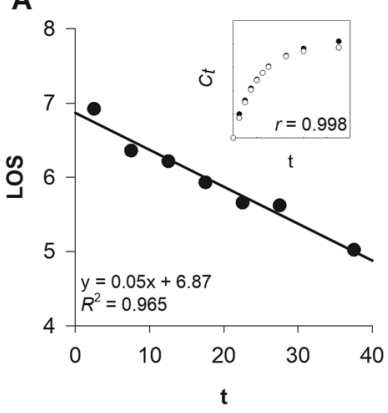

B

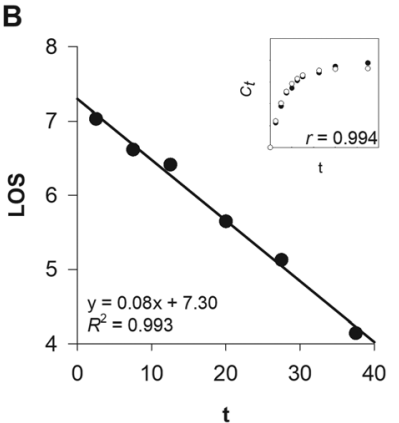

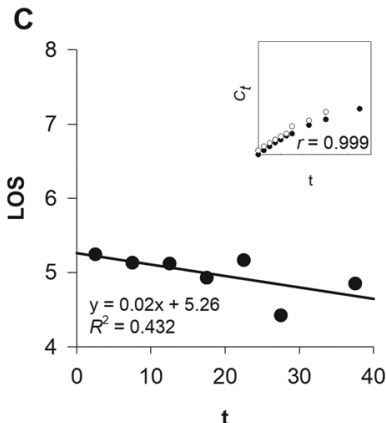

Fig. 3 Logarithm of Slope (LOS) plots for Cornflakes (A), Cream Crackers (B) and Brown Lentils (C). Inserts show calculated (filled circles) and experimental (open circles) data values for maltose concentration $\left(C_{t}\right)$ over time $(t)$. The calculated values are obtained from the first-order equation using the $k$ and $C_{\infty}$ values obtained from the LOS plot, and $r$ (Pearson's $r$ ) is the correlation between predicted and experimental data as shown in the insert. $R^{2}$ is the co-efficient of determination obtained from linear regression in the LOS plot.

in parentheses, Table 2), as the digestion progress curves were essentially zero-order, and therefore not appropriate for $1^{\text {st }}$ order kinetic analysis. This was a limitation of the data collected rather than the LOS analysis, and the $C_{\infty}$ and $k$ values for these 6 samples were therefore excluded from subsequent statistical analysis. For the remaining food products $(n=14)$, the digestibility data followed $1^{\text {st }}$ order kinetics and was suitable for LOS analysis, and the LOS plots (Fig. 3A and B) were used to obtain the kinetic parameters $C_{\infty}$ and $k$ (see Table 2), which provided a good fit to experimental data collected at time points up to $90 \mathrm{~min}$ (Pearson's $r=\geq 0.97$ ).

An overview of the various starch digestibility indices is shown in Table 2 . The in vitro indices obtained directly from the starch digestibility curves $\left(C_{20}, C_{60}, C_{90}\right.$ and $\left.\mathrm{HI}\right)$ and the $C_{\infty}$ obtained from LOS analyses (Fig. 3) were all significantly correlated $(p<0.01)$ with literature GI values, both in terms of rank (Kendalls Tau b test) and absolute values (Pearson's correlation). Scatter plots showing the relationship between in vivo and in vitro values are shown in OSM2. Out of all the indices compared, $C_{90}$ was most strongly correlated with the absolute GI values $(r=0.724, p<0.001) . C_{90}$ and $C_{\infty}$ were the most strongly correlated with in vivo rankings for GI of matched food products $\left(T_{\mathrm{b}}=0.596, p<0.001\right.$ and $T_{\mathrm{b}}=0.599, p<0.01$, respectively). The only in vitro index that did not correlate with in vivo values was the rate constant, $k$ obtained from LOS analysis. The value of $k$ seemed to be inversely related with absolute GI values, however the relationship was not statistically significant $(r=-0.590, p>0.1)$.

Table 2 Starch digestibility indices ${ }^{a, b, c}$

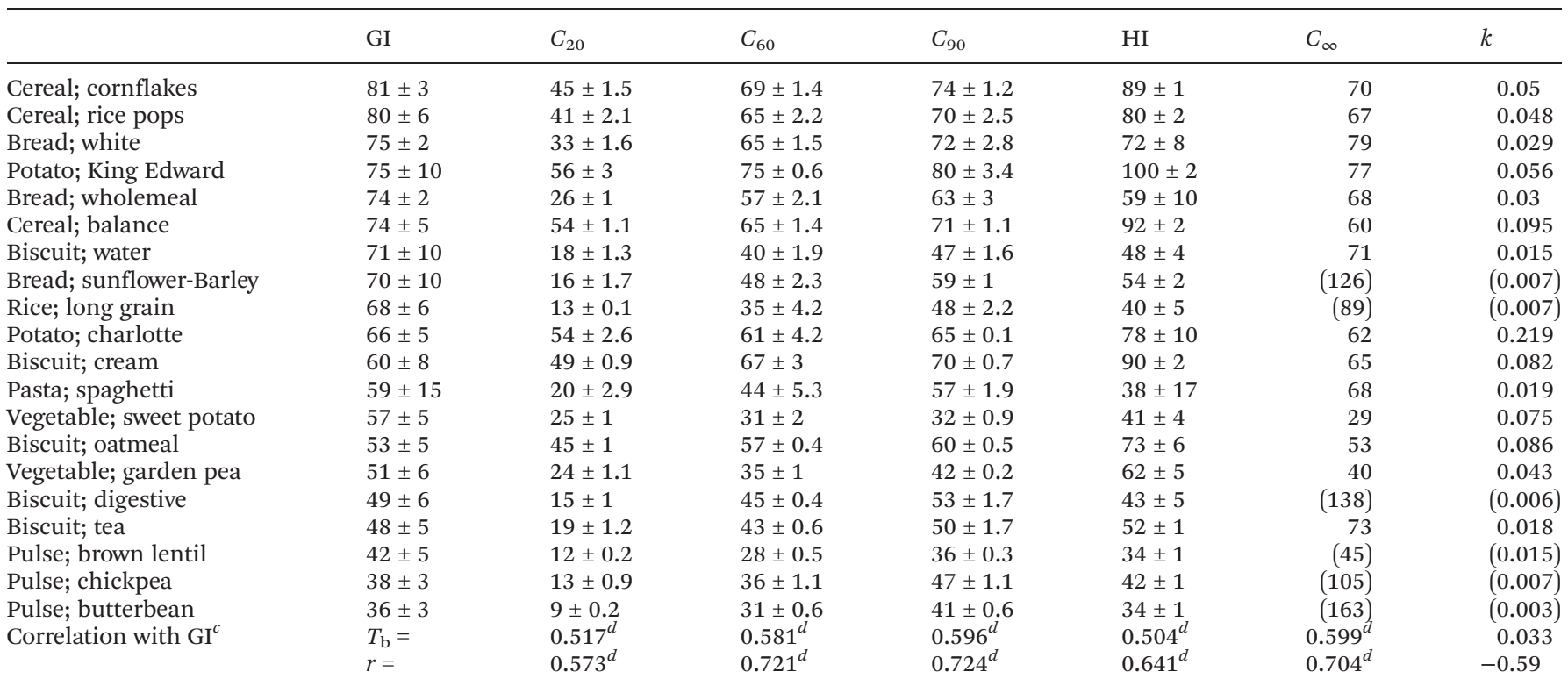

${ }^{a}$ Values are mean \pm SEM. ${ }^{b}$ Numbers in brackets were identified as outliers because the experimental data was not appropriate for LOS analysis and were therefore excluded from statistical analysis. ${ }^{c}$ Statistical tests: Kendalls Tau b, $T_{\mathrm{b}}$ and Pearson's correlation, $r .{ }^{d}$ Correlation is significant at the 0.01 level (2-tailed). 


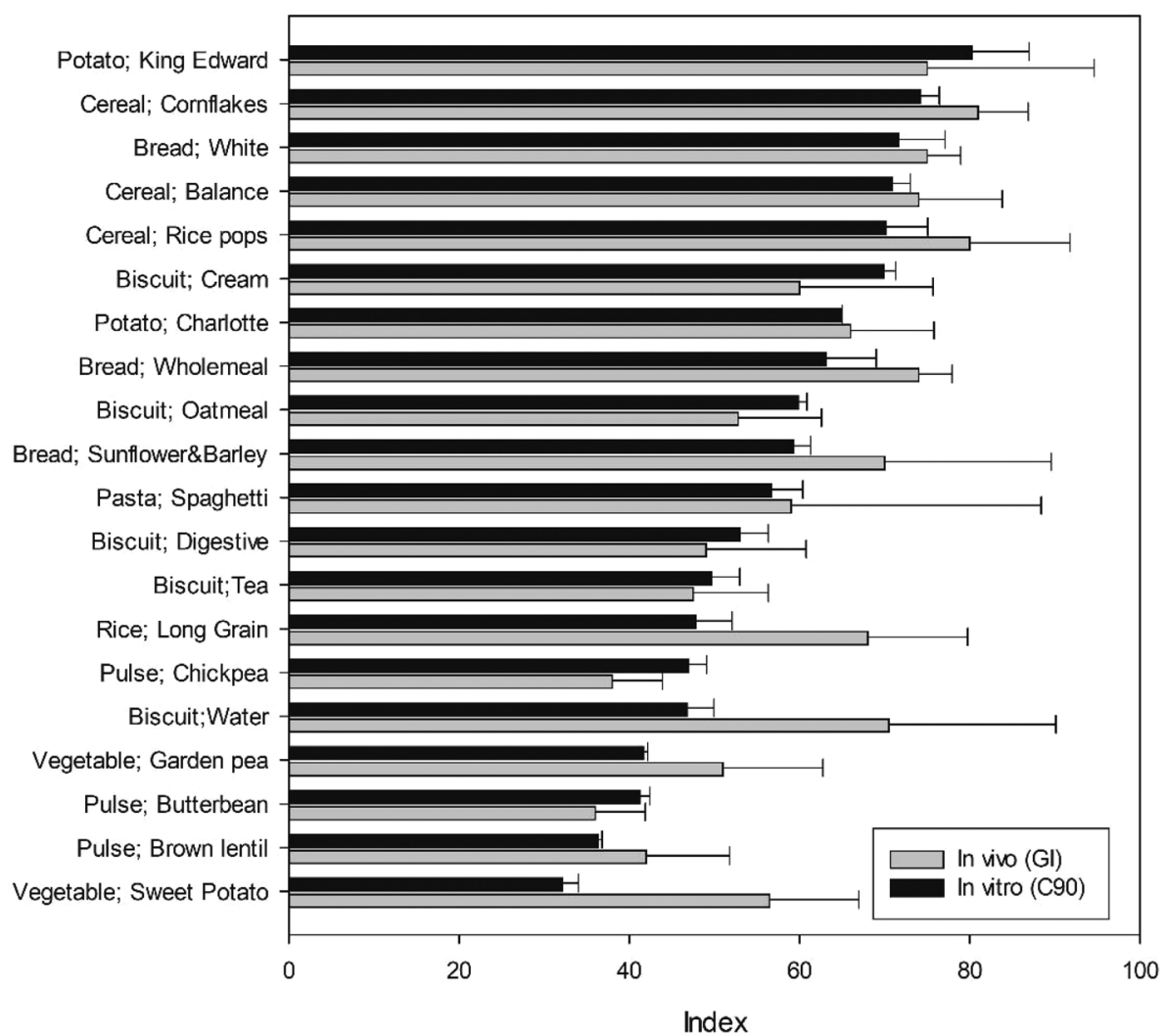

Fig. 4 In vitro values for $\mathrm{C} 90$ shown alongside in vivo Gl values. In vitro values were obtained based on $C_{90}$ and in vivo values for matched food products $(n=20)$ were taken from the published literature. All values are means shown with error bars as $95 \%$ confidence intervals.

The $C_{90}$ (which correlated most strongly with GI), obtained in vitro is shown alongside in vivo GI values for corresponding food products in Fig. 4 and together with all in vitro indices in Fig. 5. It is noteworthy that the standard deviation of in vivo measurements was greater than those observed in vitro, and the in vitro values were within the $95 \%$ confidence interval of the in vivo values, with the exception of long grain rice, chickpea, water biscuit, wholemeal bread and sweet potato. It is plausible that this is due to difference in the food material (e.g., processing, preparation, botanical source) rather than a limitation of the in vitro starch digestibility methodology per se. The in vitro $C_{90}$ values ranged from $32 \%$ to $80 \%$ starch digested, and corresponding GI values ranged from 36 to 81 .

In terms of nutrient composition (OSM1) starch was the main carbohydrate (mean $\pm \mathrm{SD}=60 \pm 18 \mathrm{~g}$ starch per $100 \mathrm{~g}$ dry weight) in $18 / 20$ foods tested in the present study, the exceptions being sweet potato and garden peas, where the sugar content was higher than the starch content. The fat content was less than $5 \%$ (dry weight basis) for $14 / 20$ foods, the exceptions being biscuits which contained up to $29 \%$ fat (dry weight basis). Similarly, protein content ranged from $5-26 \%$ of dry weight in the tested products, where the pulses and bread products were at the top end of this range. These compositional differences do not seem to systematically influence the reliability of GI predictions.

\section{Discussion}

In the present study, we used a relatively simple in vitro starch amylolysis assay to obtain indices of starch digestibility $\left(C_{20}\right.$, $C_{60}, C_{90}, \mathrm{HI}, C_{\infty}$, and $k$ ) for 20 different food products with a known GI. Despite the simplicity of the in vitro method, a reasonable correlation was observed between in vitro indices and in vivo GI values, which demonstrates that the in vitro methodology shows potential for predicting relative differences in in vivo GI. Overall, the proposed assay methodology was well-suited to the broad range of starch-rich food products tested, and the low standard error and high sensitivity enabled differences in starch digestibility profiles of similar food products to be detected.

Starch amylolysis has long been known to influence glycaemic responses, and several studies have reported on such correlations previously. ${ }^{4,7,17,18}$ Out of all the indices tested in the present study, the extent of starch digested at $90 \mathrm{~min}\left(C_{90}\right)$ was most strongly correlated with GI and for most foods the in vitro value was within the range of in vivo values for matched food products. Reasonable predictions were achieved for products from all product categories. Notable exceptions were sweet potato and garden pea, in which the high sugar content (48 and $25 \mathrm{~g}$ sugar per $100 \mathrm{~g}$ dry weight) is likely to be contributing to the GI observed in vivo. The in vitro method also seemed to underestimate the GI of water biscuits, however 


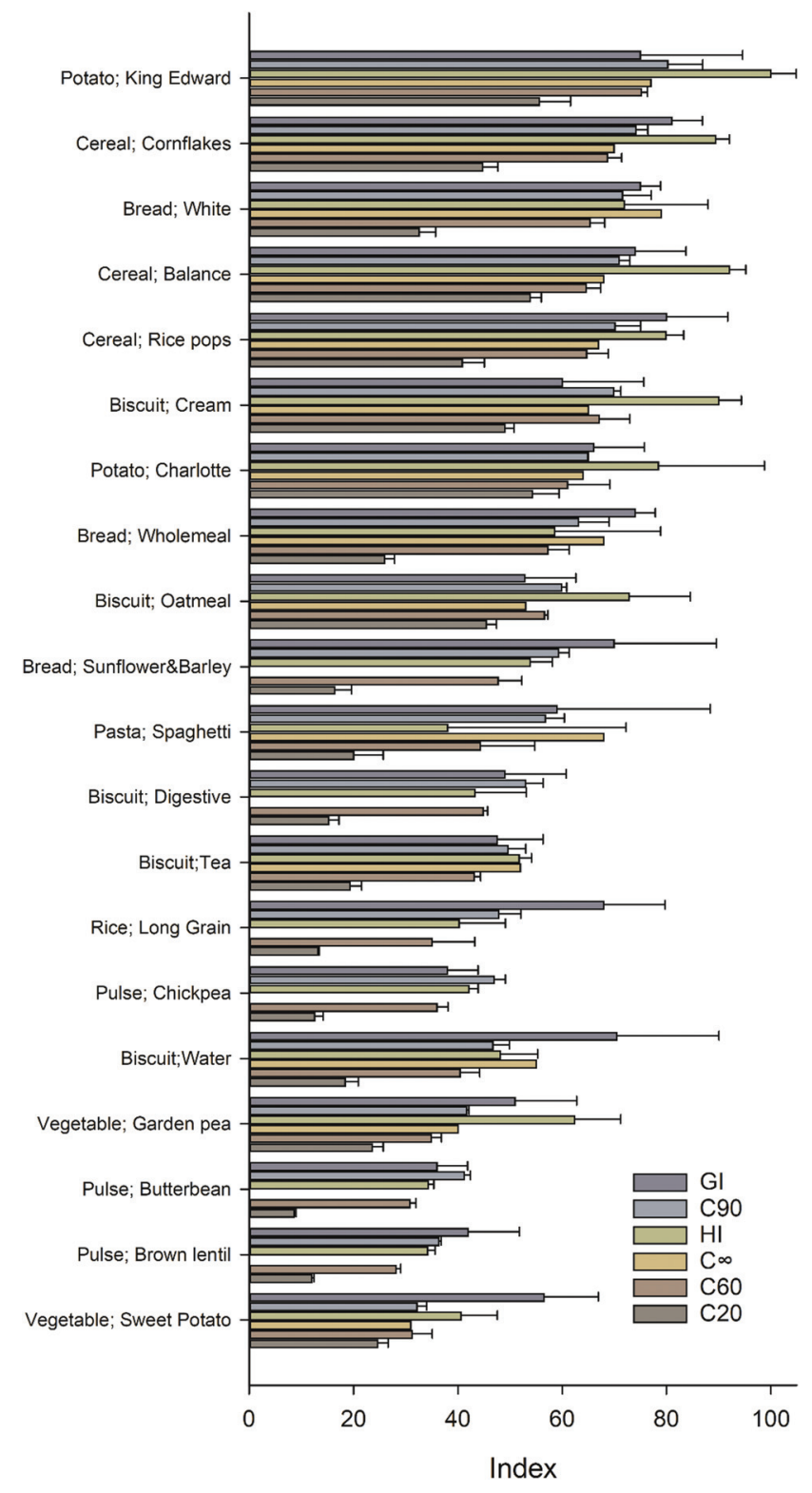

Fig. 5 All in vitro indices shown alongside in vivo $\mathrm{Gl}$ values. In vitro values for $C_{90}, \mathrm{HI}, C_{\infty}, C_{60}$ and $C_{20}$ obtained from analysis of starch digestibility data and in vivo values for matched food products were taken from the published literature. Values are shown for all 20 food products, with the exception of $C_{\infty}$ which could not be obtained for some products (no bar shown for missing values). All values are means shown with error bars as $95 \%$ confidence intervals.

the product analysed in vitro was from a different manufacturer than those used in the in vivo studies, and the products that were compared may have had different characteristics. The GI values for long grain rice and wholemeal bread were also higher than the in vitro predictions. These products are particularly susceptible to retrogradation, and a difference in how these foods were handled and prepared for testing could explain this discrepancy. There was however, also a high variability in GI values reported for many of the food products, which may reflect inconsistencies in the characteristics of the foods used. For example, cooking and cooling profiles are well known to have a major impact on starch structure and digestion kinetics in foods such as spaghetti, rice and potato ${ }^{11,19-21}$ and are a likely source of variability in GI values. A strength of the in vitro methodology is that it provides for systematic studies of such parameters and can thereby be used to understand the consequences of these factors, prior to in vivo trials.

We previously reported on the value of using LOS analysis of digestibility plots for mechanistic understanding of factors underpinning differences in starch digestibility ${ }^{12,13}$ and have recently applied this technique to examine the effect of particle size, cell wall encapsulation and starch structure on digestion kinetics. ${ }^{12,15,22}$ Within the present study we considered the use of $C_{\infty}$ and $k$ (obtained from LOS plots) as a potential predictive index of GI. The theoretical basis for this was that $C_{\infty}$ reflects the proportion of starch that has the potential to be digested over an infinite time period and thereby resembles the potential glycaemic load, whereas $k$ provides an indication of the rate at which starch-digestion products become available for absorption. In our dataset, the value of $C_{\infty}$ was reasonably correlated with GI values, however the value of the rate constant was not. This suggests that GI cannot be predicted from the rate constant alone, but requires both the rate and extent of digestion to be taken into account.

For many of the food products tested, the $C_{\infty}$ values were similar to $C_{90}$, meaning that the amylolysis reaction was nearing completion within the $90 \mathrm{~min}$ of the assay. This occurred mainly for the more rapidly digested products with a medium to high GI, and for these products $C_{\infty}$ and $C_{90}$ both provided a reasonable estimation of GI. Obtaining reliable estimates of $C_{\infty}$ for some slowly digested foods (such as pulses, digestive biscuits and long grain rice) proved more challenging because the digestibility plots were linear and therefore unsuitable for LOS analyses. Although this limitation could be overcome by using a higher enzyme-substrate ratio for in vitro assays on low GI foods, it is unclear how relevant $C_{\infty}$ values would be to prediction of GI under these circumstances. The value of $C_{\infty}$ reflects the endpoint of starch amylolysis if it is allowed to progress over infinite time. However, in vivo the duration of starch exposure to pancreatic $\alpha$-amylase in the intestinal lumen would occur over a limited time period. ${ }^{23}$ Thus, $C_{\infty}$ might be expected to overestimate the glycaemic potency of starch in some slowly digested products. In vivo, the proportion of starch that escapes digestion in the upper gastrointestinal tract would be physiologically classed as resistant starch. Thus, the proportion of starch that has not been digested by $\alpha$-amylase after a fixed time period can be considered as 'potentially resistant' starch, ${ }^{7}$ although the relationship between this in vitro indicator of resistant starch and the quantity of resistant starch that enters the colon has yet to be investigated.

In recent years, in vitro digestion models have become more advanced to provide a more physiological representation of digestive conditions, and now typically include an oral, gastric and intestinal phase to mimic the changing biochemical conditions (enzymic, pH) encountered during digestive 
passage. ${ }^{5,24}$ In the present study, however, there was no oral or gastric phase prior to amylolysis, only one enzyme (porcine pancreatic $\alpha$-amylase) was used and under a fixed enzyme-substrate ratio. This protocol resembled an enzyme-kinetic experiment rather than a simulation of physiological digestive conditions, with the advantage that low standard errors can be achieved. Overall, this is a user-friendly protocol with potential for high-throughput screening.

Users of sophisticated digestion models may be surprised by the predictive power of the 'enzyme-kinetic' approach used in this study. There may be an expectation that the inclusion of proteases and lipases is necessary to obtain a reliable view of starch digestion in real foods. One key consideration is that for many starch-rich staple foods, including most of those tested in the present study, the primary digestible component is starch. The starch digestibility screening method used in the present study is based on the premise that amylolysis is the rate-limiting step in the digestion process, and therefore has a major impact on glucose availability from food. Factors that are known to have a major impact on starch-susceptibility to digestion and thereby GI include processing conditions (i.e. which impact on starch molecular organisation $)^{25-27}$ and physical properties of the food matrix (e.g., particle size and permeability to digestive enzymes). ${ }^{9,10,15,18,28,29}$ Some properties (e.g., starch crystallinity) can be reproduced by subjecting food materials to realistic cooking/processing conditions. Particle size and microstructure however is more challenging to reproduce in vitro. In vivo mastication produces a range of particle size distributions and significant variations exist between individuals and between food materials tested. ${ }^{30}$ With the view of providing a starch digestibility screening tool and its likely applications, our preferred approach in this study was to control and standardise food structures used in the digestion. Particles were generally ground or sieved to a fixed size to preserve micro-scale structures and plant cells, although it is noteworthy that highly processed and refined starch-rich foods may not contain this level of structural integrity. This approach is more mechanistic than realistic, and users are encouraged to tailor the approach to address their research question.

One limitation of this study is that the in vitro and in vivo values were not obtained from exactly the same food products. As discussed, subtle differences in food preparation or composition can influence the digestibility and GI of foods. It remains possible that some of the observed discrepancies between in vitro and in vivo values were due to differences in the characteristics of the foods tested, rather than a limitation of the in vitro assay methodology.

Human GI studies are relatively expensive and time-consuming, and do not provide for a large number of products to be tested. Nevertheless, this study provides justification for a future validation study, in which a representative selection of well-characterised foods can be tested in parallel in vitro and in vivo.

Understanding the critical factors driving the rate of starch digestion in foods will help to inform and improve the design of food preparation protocols for in vitro testing and may also aid researchers in identifying the most suitable protocol for addressing specific research questions. Although the singleenzyme (i.e. amylase only) system used in the present study provided reasonable predictions of the glycaemic index, it would not be expected to perform as well for foods where antinutritional inhibitors of glucose absorption are rate-limiting, or where co-digestion of fat and/or protein is necessary to expose starch to digestive fluids. It is currently unclear to what extent these mechanisms apply in different food products. Further investigations are needed to improve understanding of the mechanisms and rate-limiting steps that govern digestion of food and metabolic responses and this will require a combination of in vitro and in silico digestion models and in vivo data.

\section{Conclusions}

The in vitro method presented in this study provides for rapid and accurate comparison of starch digestibility and/or resistance. Starch digestibility indices obtained in vitro are significant correlated to GI values for matched food products and show potential for use as predictive indicators of GI. We envisage that the simple protocol presented will serve as a high throughput screening method to precede and complement more advanced in vitro and in silico digestion models and in vivo studies.

\section{Conflicts of interest}

There are no conflicts of interest to declare.

\section{Acknowledgements}

The author(s) gratefully acknowledge the support of the Biotechnology and Biological Sciences Research Council (BBSRC); this research was funded by the BBSRC Institute Strategic Programme Food Innovation and Health BB/ R012512/1 and its constituent projects BBS/E/F/000PR10345 (Theme 2, Digestion in the Upper GI Tract) and BBS/E/F/ 000PR10343 (Theme 1, Food Innovation).

\section{References}

1 D. J. A. Jenkins, C. W. C. Kendall, L. S. A. Augustin, S. Franceschi, M. Hamidi, A. Marchie, A. L. Jenkins and M. Axelsen, Glycemic index: Overview of implications in health and disease, Am. J. Clin. Nutr., 2002, 76, 266S-273S.

2 F. Brouns, I. Bjorck, K. N. Frayn, A. L. Gibbs, V. Lang, G. Slama and T. M. S. Wolever, Glycaemic index methodology, Nutr. Res. Rev., 2008, 18, 145-171.

3 F. S. Atkinson, K. Foster-Powell and J. C. Brand-Miller, International Tables of Glycemic Index and Glycemic Load Values: 2008, Diabetes Care, 2008, 31, 2281-2283. 
4 D. J. A. Jenkins, H. Ghafari, T. M. S. Wolever, R. H. Taylor, A. L. Jenkins, H. M. Barker, H. Fielden and A. C. Bowling, Relationship between rate of digestion of foods and postprandial glycaemia, Diabetologia, 1982, 22, 450-455.

5 M. Minekus, M. Alminger, P. Alvito, S. Ballance, T. Bohn, C. Bourlieu, F. Carriere, R. Boutrou, M. Corredig, D. Dupont, C. Dufour, L. Egger, M. Golding, S. Karakaya, B. Kirkhus, S. Le Feunteun, U. Lesmes, A. Macierzanka, A. Mackie, S. Marze, D. J. McClements, O. Menard, I. Recio, C. N. Santos, R. P. Singh, G. E. Vegarud, M. S. Wickham, W. Weitschies and A. Brodkorb, A standardised static in vitro digestion method suitable for food - an international consensus, Food Funct., 2014, 5, 1113-1124.

6 A. Guerra, L. Etienne-Mesmin, V. Livrelli, S. Denis, S. Blanquet-Diot and M. Alric, Relevance and challenges in modeling human gastric and small intestinal digestion, Trends Biotechnol., 2012, 30, 591-600.

7 I. Goñi, A. Garcia-Alonso and F. Saura-Calixto, A starch hydrolysis procedure to estimate glycemic index, Nutr. Res., 1997, 17, 427-437.

8 K. N. Englyst, H. N. Englyst, G. J. Hudson, T. J. Cole and J. H. Cummings, Rapidly available glucose in foods: an in vitro measurement that reflects the glycemic response, Am. J. Clin. Nutr., 1999, 69, 448-454.

9 M. M. L. Grundy, C. H. Edwards, A. R. Mackie, M. J. Gidley, P. J. Butterworth and P. R. Ellis, Re-evaluation of the mechanisms of dietary fibre and implications for macronutrient bioaccessibility, digestion and postprandial metabolism, Br. J. Nutr., 2016, 116, 816-833.

10 J. Singh, A. Dartois and L. Kaur, Starch digestibility in food matrix: a review, Trends Food Sci. Technol., 2010, 21, 168-180.

11 J. Parada and J. M. Aguilera, Review: Starch matrices and the glycemic response, Food Sci. Technol. Int., 2011, 17, 187-204.

12 C. H. Edwards, F. J. Warren, P. J. Milligan, P. J. Butterworth and P. R. Ellis, A novel method for classifying starch digestion by modelling the amylolysis of plant foods using firstorder enzyme kinetic principles, Food Funct., 2014, 5, 27512758.

13 P. J. Butterworth, F. J. Warren, T. Grassby, H. Patel and P. R. Ellis, Analysis of starch amylolysis using plots for first-order kinetics, Carbohydr. Polym., 2012, 87, 2189-2197.

14 Public Health England (2019) McCance \& Widdowson's composition of foods integrated dataset. Available online: https://www.gov.uk/government/publications/compositionof-foods-integrated-dataset-cofid.

15 C. H. Edwards, M. Maillot, R. Parker and F. J. Warren, A comparison of the kinetics of in vitro starch digestion in smooth and wrinkled peas by porcine pancreatic alphaamylase, Food Chem., 2018, 244, 386-393.

16 M. Lever, A new reaction for colorimetric determination of carbohydrates, Anal. Biochem., 1972, 47, 273-279.
17 Y. Granfeldt, I. Bjorck, A. Drews and J. Tovar, An in vitro procedure based on chewing to predict metabolic response to starch in cereal and legume products, Eur. J. Clin. Nutr., 1992, 46, 649-660.

18 K. A. Germaine, S. Samman, C. G. Fryirs, P. J. Griffiths, S. K. Johnson and K. J. Quail, Comparison of in vitro starch digestibility methods for predicting the glycaemic index of grain foods, J. Sci. Food Agric., 2008, 88, 652-658.

19 S. Wang and L. Copeland, Molecular disassembly of starch granules during gelatinization and its effect on starch digestibility: A review, Food Funct., 2013, 4, 1564-1580.

20 J. Parada and J. M. Aguilera, In vitro Digestibility and Glycemic Response of Potato Starch is Related to Granule Size and Degree of Gelatinization, J. Food Sci., 2009, 74, E34-E38.

21 M. Stuknyte, S. Cattaneo, M. A. Pagani, A. Marti, V. Micard, J. Hogenboom and I. De Noni, Spaghetti from durum wheat: effect of drying conditions on heat damage, ultrastructure and in vitro digestibility, Food Chem., 2014, 149, 40-46.

22 H. Patel, R. Day, P. J. Butterworth and P. R. Ellis, A mechanistic approach to studies of the possible digestion of retrograded starch by alpha-amylase revealed using a log of slope (LOS) plot, Carbohydr. Polym., 2014, 113, 182-188.

23 P. Layer and G. Gröger, Fate of Pancreatic Enzymes in the Human Intestinal Lumen in Health and Pancreatic Insufficiency, Digestion, 1993, 54(suppl 2), 10-14.

24 J. W. Woolnough, J. A. Monro, C. S. Brennan and A. R. Bird, Simulating human carbohydrate digestion in vitro: a review of methods and the need for standardisation, Int. J. Food Sci. Technol., 2008, 43, 2245-2256.

25 R. F. Tester and W. R. Morrison, Swelling and gelatinization of cereal starches. I. Effects of amylopectin, amylose, and lipids, Cereal Chem., 1990, 67, 551-557.

26 C. H. Edwards, F. J. Warren, G. M. Campbell, S. Gaisford, P. G. Royall, P. J. Butterworth and P. R. Ellis, A study of starch gelatinisation behaviour in hydrothermally-processed plant food tissues and implications for in vitro digestibility, Food Funct., 2015, 6, 3634-3641.

27 S. M. Kingman and H. N. Englyst, The influence of food preparation methods on the in vitro digestibility of starch in potatoes, Food Chem., 1994, 49, 181-186.

28 S. Dhital, R. R. Bhattarai, J. Gorham and M. J. Gidley, Intactness of cell wall structure controls the in vitro digestion of starch in legumes, Food Funct., 2016, 7, 1367-1379.

29 A. Lovegrove, C. H. Edwards, I. De Noni, H. Patel, E. Sedef, T. Grassby, C. Zielke, M. Ulmius, L. Nilsson, P. J. Butterworth, P. R. Ellis and P. R. Shewry, Role of Polysaccharides in Food, Digestion and Health, Crit. Rev. Food Sci. Nutr., 2017, 57, 237-253.

30 G. M. Bornhorst and R. P. Singh, Bolus formation and disintegration during digestion of food carbohydrates, Compr. Rev. Food Sci. Food Saf., 2012, 11, 101-118. 